\title{
Prognostic impact of residual SYNTAX score in patients with obstructive sleep apnea and acute coronary syndrome: a prospective cohort study
}

\author{
Yaping Zeng ${ }^{1+}$, Shuhan Yang ${ }^{1,2+}$, Xiao Wang ${ }^{1}$, Jingyao Fan ${ }^{1}$, Shaoping $\mathrm{Nie}^{1 *}$ and Yongxiang Wei ${ }^{3^{*}}$
}

\begin{abstract}
Background: There is a paucity of data from large prospective study evaluating the prognostic significance of the residual Synergy between percutaneous intervention with Taxus drug-eluting stents and cardiac surgery (SYNTAX) Score (rSS) in patients with obstructive sleep apnea (OSA) and Acute Coronary Syndrome (ACS).

Methods: ACS patients who undergoing percutaneous coronary angiography and completing a sleep study during hospitalization were prospectively enrolled. The baseline SYNTAX Score (bSS) and the rSS after revascularization were assessed. Complete revascularization ( $C R, r S S=0)$ and incomplete revascularization $(I C R, r S S>0)$ were categorized. OSA (apnea hypopnea index, $\mathrm{AHI} \geq 15$ ) and non-OSA $(\mathrm{AHI}<15)$ were grouped according to $\mathrm{AHI}$. The primary endpoint of the study was major adverse cardiovascular and cerebrovascular events (MACCEs), defined as a composite of cardiovascular death, myocardial infarction, stroke, ischemia-driven revascularization, or hospitalization for UAP or heart failure.

Results: Overall, 752 patients were prospectively enrolled. At a median follow-up of 1 year, the incidence of MACCEs was significantly higher in the OSA than in the non-OSA group (hazard ratio [HR]:1.68; 95\% confidence interval [CI]:1.04-2.72; $P$ $=.034$ ). ICR was associated with a higher risk of MACCEs in the non-OSA group (HR:3.34;95\% Cl:1.0-11.12; $P=.05$ ). The OSA patients with ICR had a 5.1 higher risk of MACCEs compared with the non-OSA with CR group, $P=.007$. The OSA patients with CR had a similar 1-year MACCEs as all the non-OSA patients (HR:1.10; 95\% Cl:0.515-2.349; $P=0.806$ ).
\end{abstract}

Conclusions: ACS patients with OSA and ICR have a high rate of MACCEs at 1 year. In contrast, the prognosis of ACS patients with OSA but CR is favorable and similar to patients without OSA. Adequate level of revascularization is recommended to optimize clinical outcomes in ACS patients with OSA.

Trial registration: Clinicaltrials.gov identifier NCT03362385.

Keywords: Obstructive sleep apnea, Residual SYNTAX score, Acute coronary syndrome

\section{Backgrounds}

OSA (Obstructive Sleep Apnea) characterized by repeated hypoxemia at night, may promote the occurrence and development of cardiovascular disease through the activation of sympathetic system, inflammatory reaction,

\footnotetext{
*Correspondence: spnie@ccmu.edu.cn; weiyongxiang@vip.sina.com ${ }^{\dagger}$ Yaping Zeng and Shuhan Yang contributed equally to this work ${ }^{1}$ Emergency \& Critical Care Center, Beijing Anzhen Hospital, Capital Medical University, 2nd Anzhen Road, Chaoyang District, Beijing 100029, China ${ }^{3}$ Department of Otolaryngology Head \& Neck Surgery, Beijing Anzhen Hospital, Capital Medical University, 2nd Anzhen Road, Chaoyang District, Beijing 100029, China

Full list of author information is available at the end of the article
}

oxidative stress and metabolic abnormalities [1-3]. OSA has been advocated as a new emerging independent risk factor for coronary artery disease by America expert consensus documents and Europe clinical guidelines [4-6]. It has been confirmed recently in a large prospective cohort study [7]. Intravascular Ultrasound has further demonstrated those patients with OSA present greater plaque burden [8]. However, the relation between cardiovascular outcomes and OSA still remain debatable even in randomized trials $[4,9,10]$.

The Synergy between percutaneous intervention with Taxus drug-eluting stents and cardiac surgery (SYNTAX)

(c) The Author(s). 2019 Open Access This article is distributed under the terms of the Creative Commons Attribution 4.0 International License (http://creativecommons.org/licenses/by/4.0/), which permits unrestricted use, distribution, and 
score has been developed to stratify the complexity and severity of coronary lesions [11]. Residual SYNTAX Score (rSS) was derived from SYNTAX score to quantify the burden of residual coronary artery disease after percutaneous coronary intervention (PCI) or coronary artery bypass graft (CABG), and has been validated as an independent predictor for clinical adverse events [12-14]. However, studies examining the impact of rSS on patients with OSA and acute coronary syndrome (ACS) have not yet been elucidated.

The objective of the present study was to evaluate the prognostic impact with rSS in patients with OSA and ACS in a prospective single center cohort study.

\section{Methods and materials}

\section{Study design and subjects}

The OSA-ACS project (NCT03362385) was a prospective, single-center, observational cohort study that recruited consecutive patients with ACS diagnosis at Beijing An Zhen Hospital, Capital Medical University, Beijing, China from May 2015 to June 2017. A total of 899 eligible ACS patients who have undergone overnight portable sleep study were eligible for inclusion. ACS includes ST-segment elevation myocardial infarction (STEMI), none ST-segment elevation myocardial infarction (NSTEMI), and unstable angina pectoris (UAP). Inclusion criteria: i) age $\geq 18$ years old and $<85$ years old. ii) ACS. Exclusion criteria: i) cardiogenic shock; ii) cardiac arrest; iii) history of malignancy; iv) failed sleep study; v) failure of coronary angiography or poor quality; vi) central sleep apnea; vii) known OSA and continuous positive air pressure (CPAP) treatment. The study was approved by the Institutional Review Board of Beijing Anzhen Hospital, Capital Medical University and all patients provided written informed consent.

\section{Overnight level 3 polygraphy and definitions}

The sleep studies were conducted with a portable diagnostic device (Apnealink Air, Resmed, Australia) for more than $7 \mathrm{~h}$ during the night after admission by a trained research team. Nasal airflow, thoracic-abdominal movements, arterial oxygen saturation, heart rate, and snoring episodes were recorded. According to apnea hypopnea index (AHI), patients were categorized to 2 groups: OSA group (AHI $\geq 15$ events $/ \mathrm{h}$ ), non-OSA group (AHI $<15$ events/h). For the patients with moderate to severe sleep apnea $(\mathrm{AHI} \geq 15)$, particularly those with excessive daytime sleepiness, we referred them to sleep center for further evaluation and consideration of CPAP therapy.

\section{Procedure}

The decision of PCI strategy and stenting were left to the discretion of the operator. Dual antiplatelet therapy with aspirin and $\mathrm{P}^{2} \mathrm{Y}_{12}$ receptor inhibitor (i.e. clopidogrel or ticagrelor) were recommended for at least 12 months according to the current standard guidelines. All patients were followed up routinely in outpatients or by phone at 1 month, 3 months, 6 months, 12 months, and every 6 months thereafter.

\section{SYNTAX SCORE assessment}

Basic SYNTAX Score (bSS) and rSS were calculated by 2 experienced interventional cardiologists who were blinded to the clinical outcomes and the sleep patterns of the patients using a web-based calculator (www.syntaxscore.com), and disagreement was resolved by consensus. Lesions with $>50 \%$ diameter stenosis in vessel $\geq 1.5 \mathrm{~mm}$ in diameter were scored using the SS algorithm described previously.

The rSS was defined as the remaining SYNTAX Score after PCI or CABG. In the case of staged PCI procedures, the final planned procedure was used to calculate the point for rSS. For the patients who underwent CABG, the rSS was scored by comparing the bSS with the surgical procedure reports, the same, the final decision was made by consensus in cases of disagreement between the 2 cardiologists. Moreover, each vessel disease was still scored in terms of vessel characteristics (tortuosity, severe calcification) or any residual lesions distal to the graft allocation [14]. Patients were then classified into 2 groups based on the points of rSS: complete revascularization $(\mathrm{CR})$ : $\mathrm{rSS}=0$; incomplete revascularization (ICR): $\mathrm{rSS}>0$ [15].

The intra-observer variability of the bSS and rSS using the intraclass correlation analysis were $1.0(0.99-1.0), P$ $<.001$ and 0.98 (0.95-0.99), $P<.001$. The inter-observer variability of the bSS and rSS was $1.0(0.99-1.0)$ and 0.99 (0.99-0.99) respectively, both $P<.001$.

\section{The primary endpoints and definitions}

The primary endpoint of the study was major adverse cardiovascular and cerebrovascular events (MACCEs), defined as a composite of cardiovascular death, myocardial infarction, stroke, ischemia-driven revascularization, or hospitalization for UAP or heart failure.

\section{Statistical analysis}

Continuous data were expressed as mean \pm standard deviation (SD) and compared using the Student $t$ test. The chi-square or Fisher's exact test was used for comparison of categorical variables. Clinical outcomes were determined using Kaplan-Meier method and compared using the log-rank test. Cox multivariate regression analysis was used to determine the independent predictor of clinical events, with variable entry/stay criteria of 0.1 / 0.1. Interclass correlation coefficient (ICC) was used to assess the inter-observer (Zeng $\mathrm{Y}$ and Yang $\mathrm{S}$ ) and 
intra-observer agreement of bSS, as well as rSS after PCI or CABG with $95 \%$ confidence intervals. An ICC $<0.4$ indicates bad agreement, an ICC between 0.4 and 0.75 indicates moderate agreement, and ICC values $>0.75$ indicates excellent agreement [16]. A $P$ value $<.05$ were considered statistically significant. Statistical analyses were performed using SPSS version 22 (IBM Corp, Armonk, NY).

\section{Results}

\section{Study population and case selection}

The study flowchart was shown in Fig. 1. From May 2015 to June 2017, 752 eligible patients were prospectively included in the analysis. Overall, mean age was $57.2 \pm 10.2$ years, $82.6 \%$ were male and $30.6 \%$ were diabetes. STEMI, NSTEMI, UAP according to the Braunwald classification were the clinical presentation in 32.3, 25.1 and $42.6 \%$ of the population respectively. The mean bSS and rSS was $14.6 \pm 11.4$ and $7.6 \pm 8.7$.

The cohort's baseline demographic and clinical characteristics were listed in Table 1. OSA was observed in $49.6 \%$ of the population. Mean oxygen saturation $\left(\mathrm{SO}_{2}\right)$ in the OSA $(93.0 \pm 2.3 \%)$ was lower than in the non-OSA group (94.6 $\pm 1.7 \%, P<.001)$. Interestingly, multi-vessel diseases were more frequently observed in the OSA than in the non-OSA cases (three vessel disease: $27.3 \%$ vs $22.7 \%$; two vessel disease: $38.3 \%$ vs $32.2 \%$, overall $P=.024$ ). Similarly, chronic total occlusion lesions (CTO) in the OSA (23.6\%) was more than in the non-OSA group (15.3\%), $P=.004$. Finally, more stents $(1.0 \pm 1.0)$ were implanted in the OSA than in the non-OSA group $(0.8 \pm 1.0), P=.046$.
The correlation and distribution of the bSS and the rSS The correlation and distribution of the bSS and the rSS were showed in Fig. 2a. The bSS was strongly positively correlated with the rSS $(\mathrm{R}=0.72, p<0.001)$. The frequency of patients with ICR increased across the bSS, while the frequency of CR decreased across the bSS.

\section{Clinical outcomes}

The mean follow-up was 1 year. The total MACCEs rate was significantly higher in the OSA than in the non-OSA patients $(12.3 \%$ vs $6.9 \%$; hazard ratio [HR]: 1.68; $95 \%$ confidence interval [CI]: 1.04-2.72; $P=.034$ ). However, there were no difference in the rate of ischemia-driven revascularization between the OSA and non-OSA groups $(16,4.3 \%$ vs $10,2.6 \%, p=0.215)$.

Table 2 showed the different clinical outcomes at follow-up for subgroups of the rSS. ICR was associated with a higher risk of MACCEs in the non-OSA group (HR: 3.34; 95\% CI: $1.0-11.12 ; P=.05)$. ICR had a 5.1 higher risk of MACCEs at follow-up compared with the non-OSA with CR group, $P=.007$. Figure 3 demonstrated that the OSA patients with CR $(9 / 104,8.7 \%)$ had a higher 1-year MACCEs compared to the non-OSA and CR patients (3/ 102, 2.5\%), (HR: 3.0; 95\% CI: 0.79-11.42; $P=0.11$ ), the $p$ value was not significant. Interestingly, the OSA patients with CR had a similar 1-year MACCEs as all the non-OSA patients (HR: 1.10; 95\% CI: 0.515-2.349; $P=0.806$ ). There was also no significant difference in the 1-year MACCEs rate in the OSA and in the non-OSA patients with CR (HR: 3.1; $95 \% \mathrm{CI}: 0.84-11.5 ; P=0.09)$. The MACCEs in all patients was mainly driven by hospitalization for UAP. In patients with OSA, the rate of hospitalization for UAP was

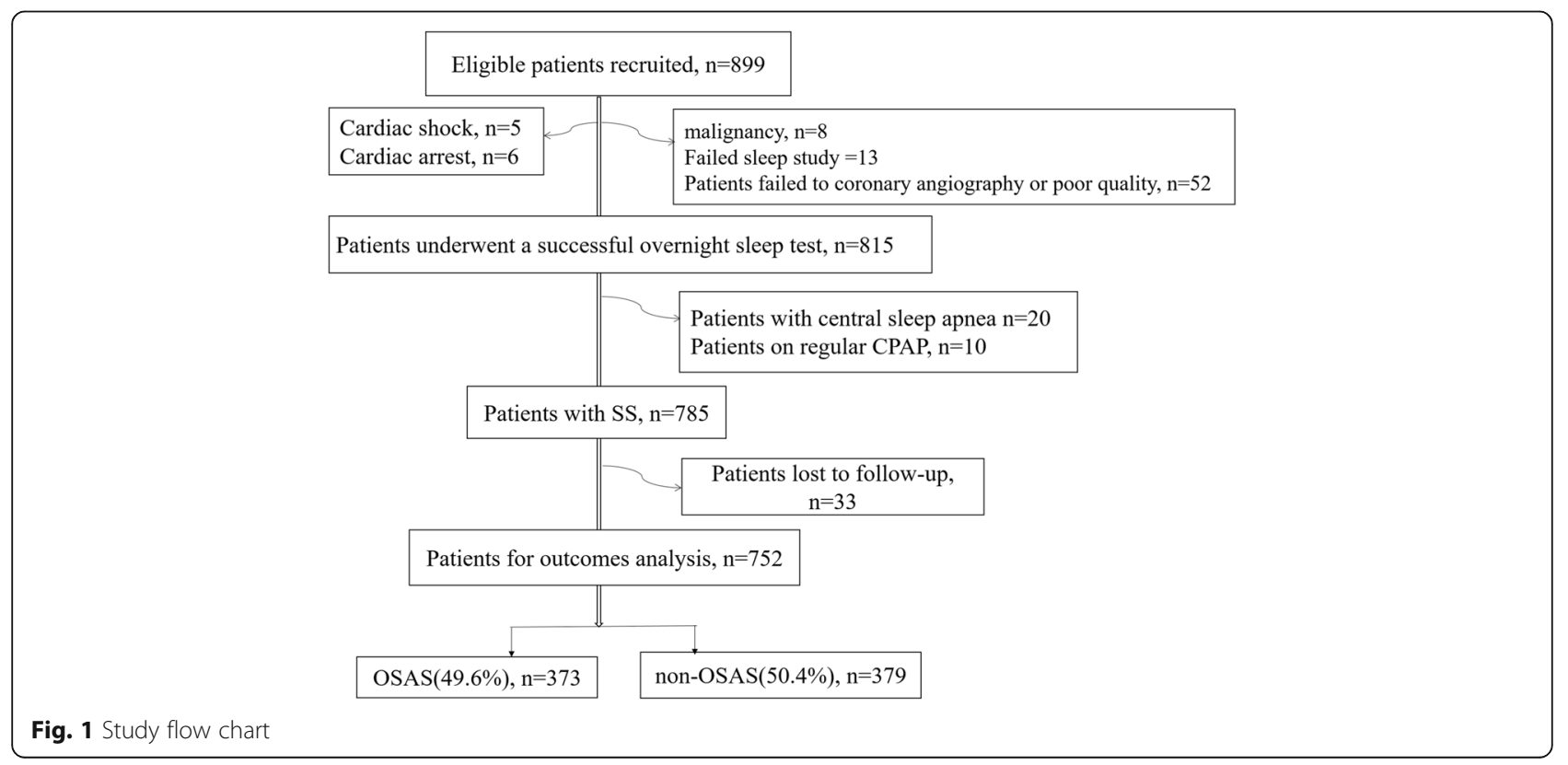


Table 1 Baseline patient characteristics

\begin{tabular}{|c|c|c|c|}
\hline & OSA $(N=373)$ & non-OSA $(N=379)$ & $P$ value \\
\hline Age, years (mean $\pm S D)$ & $57.6 \pm 10.4$ & $56.8 \pm 10.0$ & 0.299 \\
\hline Male, n (\%) & $315,84.5 \%$ & $306,80.7 \%$ & 0.211 \\
\hline BMI & $27.6 \pm 3.4$ & $25.8 \pm 3.3$ & $<0.001$ \\
\hline $\mathrm{AHI}$ & $33.7 \pm 15.1$ & $7.1 \pm 4.0$ & $<0.001$ \\
\hline bSS & $14.9 \pm 11.0$ & $14.4 \pm 11.8$ & 0.350 \\
\hline rSS & $7.8 \pm 8.3$ & $7.4 \pm 9.1$ & 0.054 \\
\hline Hypertension requiring medication, n (\%) & $255,68.4 \%$ & $239,63.1 \%$ & 0.144 \\
\hline Hyper cholesterolemia requiring medication, n (\%) & $103,27.6 \%$ & $98,25.9 \%$ & 0.621 \\
\hline Diabetes mellitus requiring medication, $\mathrm{n}(\%)$ & $112,30 \%$ & $118,31.1 \%$ & 0.752 \\
\hline Myocardial infarction history, n (\%) & $57,15.3 \%$ & $52,13.7 \%$ & 0.605 \\
\hline Cardiac Intervention history, n (\%) & $78,20.9 \%$ & $54,14.2 \%$ & 0.017 \\
\hline Time to last cardiac intervention, (days) & $57 \pm 49.6$ & $67 \pm 75$ & 0.837 \\
\hline Current smokers, n (\%) & $237,63.5 \%$ & $247,65.2 \%$ & 0.649 \\
\hline Family history of CAD, n (\%) & $31,8.3 \%$ & $26,6.9 \%$ & 0.492 \\
\hline Minimal $\mathrm{SO}_{2}, \%$ & $86.4 \pm 4.5$ & $79.4 \pm 8.6$ & $<0.001$ \\
\hline Mean $\mathrm{SO}_{2}, \%$ & $93.0 \pm 2.3$ & $94.6 \pm 1.7$ & $<0.001$ \\
\hline ODI & $33.1 \pm 16.1$ & $7.9 \pm 5.2$ & $<0.001$ \\
\hline Clinical presentation & & & 0.523 \\
\hline STEMI, n (\%) & $124,33.2 \%$ & $119,31.4 \%$ & \\
\hline NSTEMI, n (\%) & $87,23.3 \%$ & $102,26.9 \%$ & \\
\hline Unstable Angina Pectoris, n (\%) & $162,43.4 \%$ & $158,41.7 \%$ & \\
\hline LVEF, \% & $59.5 \pm 7.7$ & $59.8 \pm 8.0$ & 0.605 \\
\hline \multicolumn{4}{|l|}{ Post-procedural medication, n (\%) } \\
\hline ACE inhibitors or ARB & $271,72.7 \%$ & $258,68.1 \%$ & 0.175 \\
\hline Beta-blockers & $290,77.7 \%$ & $284,74.9 \%$ & 0.391 \\
\hline \multicolumn{4}{|l|}{ Antiplatelet therapy } \\
\hline Aspirin & $354,94.9 \%$ & $354,93.4 \%$ & 0.438 \\
\hline P2Y12 inhibitor & $342,91.7 \%$ & $331,87.3 \%$ & 0.057 \\
\hline Statin & $357,95.7 \%$ & $356,93.9 \%$ & 0.324 \\
\hline \multicolumn{4}{|l|}{ Anatomical characteristics } \\
\hline Multi-vessel disease, n (\%) & & & 0.024 \\
\hline Three vessel disease, n (\%) & $102,27.3 \%$ & $86,22.7 \%$ & \\
\hline Two vessel disease, n (\%) & $143,38.3 \%$ & $122,32.2 \%$ & \\
\hline Left main disease, n (\%) & $12,3.2 \%$ & $17,4.5 \%$ & 0.45 \\
\hline Any total occlusions & $88,23.6 \%$ & $58,15.3 \%$ & 0.004 \\
\hline Any bifurcation & $210,61.4 \%$ & $198,58.8 \%$ & 0.764 \\
\hline \multicolumn{4}{|l|}{ Any aorta-ostial lesion } \\
\hline \multicolumn{4}{|l|}{ Diffuse disease } \\
\hline Any lesion > $20 \mathrm{~mm}$ & $90,24.1 \%$ & $78,20.6 \%$ & 0.256 \\
\hline Any severe calcification & $29,7.8 \%$ & $41,10.8 \%$ & 0.168 \\
\hline Any severe tortuosity & $32,8.6 \%$ & $22,5.8 \%$ & 0.159 \\
\hline Any angiographically visible thrombus & $53,14.2 \%$ & $63,16.6 \%$ & 0.366 \\
\hline \multicolumn{4}{|l|}{ Procedure related characteristics } \\
\hline CABG, n (\%) & $34,9.1 \%$ & $41,10.8 \%$ & 0.467 \\
\hline
\end{tabular}


Table 1 Baseline patient characteristics (Continued)

\begin{tabular}{llll}
\hline & OSA $(N=373)$ & non-OSA $(N=379)$ & $P$ value \\
\hline $\mathrm{PCl}, \mathrm{n}(\%)$ & $58.7 \%$ & $51.7 \%$ & 0.057 \\
Total number of stent, $\mathrm{n}$ & $1.0 \pm 1.0$ & $0.8 \pm 1.0$ & 0.046 \\
Hospital stay, $\mathrm{n}$ & $4.9 \pm 2.9$ & $4.7 \pm 2.9$ & 0.371 \\
\hline
\end{tabular}

Values are shown in mean $\pm \mathrm{SD}$, or $\mathrm{n}(\%)$

OSA obstructive sleep apnea; BMI = body mass index, $A H I$ apnea hypopnea index, bSS baseline syntax score, $r S S$ residual syntax score, CAD coronary artery disease, Mini SO2 minimal oxygen saturation, Mean SO2 mean oxygen saturation, ODI oxygen desaturation index, STEMI ST-segment elevation myocardial infarction, NSTEMI none ST-segment elevation myocardial infarction, LVEF left ventricular ejection fraction, $A C E$ angiotensin converting enzyme, $A R B$ angiotensin receptor blocker, $C A B G$ coronary artery bypass graft, $P C l$ percutaneous coronary intervention

higher in the ICR than in the CR subgroup (HR: 6.73; 95\% CI: $1.61-28.2 ; P=.009)$. The same was also true in the non-OSA group (HR: 6.0; 95\% CI: 0.79-45.6; $P=.084$ ), although the $P$ value was not significant.
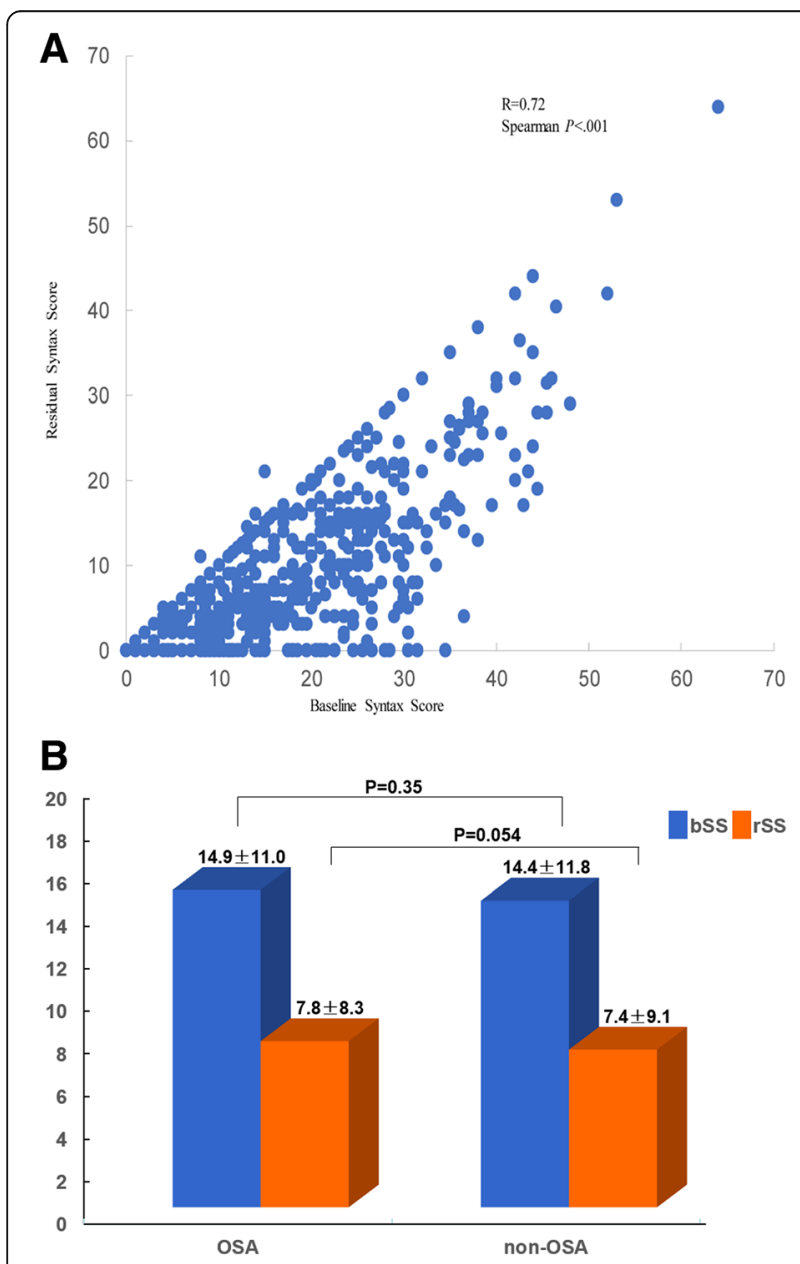

Fig. 2 a: Correlation between the baseline and the residual SYNTAX Score. Relationship between the baseline (x-axis) and residual (y-axis) SYNTAX Score after angiography in 752 patients. A strong positive correlation was present between the baseline and the residual SYNTAX Score. $\mathbf{b}$ : Column bars of the baseline and residual SYNTAX Score in the OSA and non-OSA groups. SS = SYNTAX Score; OSA $s=$ Obstructive Sleep Apnea; non-OSA = non-Obstructive Sleep Apnea

\section{Multivariate cox regression analysis}

Multivariate analysis demonstrated that OSA (HR: 1.65; 95\% CI: $1.02-2.67, P=.044$ ) and rSS (HR: 2.04; 95\% CI: 1.09-3.8, $P=.025)$ were two independent predictors of the MACCEs at follow-up (Table 3).

\section{Discussion}

The findings of the present study can be summarized as follows: 1) OSA with ACS patients were associated with a 2-fold higher risk of MACCEs than in those non-OSA; 2) compared to the non-OSA patients, OSA with ACS patients presented more severe coronary artery disease; 3) the Residual SYNTAX Score was able to stratify the OSA patients with ACS; 4) incomplete revascularization was associated with high clinical events in the OSA patients with ACS.

OSA has been advocated as a new independent risk factor for coronary artery disease [4-6]. However, the link between cardiovascular events and OSA is still controversial [10] because of the following reasons: (1) most of the studies were observational ones for both ethical and logistical reasons. Symptomatic cases will benefit from long-term CPAP, which was challenging to make OSA patients free from treatment; (2) CPAP treatment adherence of patients was important to guarantee the beneficial effect [17]. Studies showed beneficial effects of CPAP only in those who used CPAP for 4 to $6 \mathrm{~h} / \mathrm{night}$ or longer $[9,18]$; 3) OSA treatment effect might be confounded by a treatment bias, those with severe symptoms might also adhere to diet, exercise and other drugs better than those without or mild symptoms. Thus, the net beneficial effect of OSA treatment was difficult to draw [4]. Therefore, compelling causal evidences between OSA and cardiovascular events are still lacking. In the prospective study including a large Chinese ACS population in real-world, the prevalence of OSA was approximately $50 \%$, which is similar to that reported in previous studies [19]. We demonstrated that OSA was associated with a 2-fold increased risk of MACCEs compared with the non-OSA patients, which was mainly driven by unstable angina pectoris.

Elucidating the effects of OSA on the coronary angiographic characteristics is the fundamental pathologic substrate to understand the underlying effects on 
Table 2 Incidence of MACCEs stratified for residual Syntax Score groups

\begin{tabular}{|c|c|c|c|c|c|c|c|c|}
\hline & \multicolumn{4}{|l|}{ OSA $(N=373)$} & \multicolumn{4}{|c|}{ Non-OSA $(\mathrm{N}=379)$} \\
\hline & $C R(N=104)$ & ICR $(N=269)$ & $\mathrm{HR}(95 \% \mathrm{Cl})$ & $P$ value & $C R(N=120)$ & ICR $(N=259)$ & $\mathrm{HR}(95 \% \mathrm{Cl})$ & $P$ value \\
\hline Cumulative MACCEs, n (\%) & $9,8.7 \%$ & $37,13.8 \%$ & $1.77(0.85,3.67)$ & 0.126 & $3,2.5 \%$ & $23,8.9 \%$ & $3.34(1.0,11.12)$ & 0.05 \\
\hline Cardiovascular death, n (\%) & $2,1.9 \%$ & $1,0.4 \%$ & - & 0.177 & 0 & $5,1.9 \%$ & - & 0.385 \\
\hline Myocardial infarction, n (\%) & 0 & $4,1.5 \%$ & - & 0.447 & $2,1.7 \%$ & $4,1.5 \%$ & - & 0.866 \\
\hline Stroke, n (\%) & $3,2.9 \%$ & $2,0.7 \%$ & - & 0.154 & 0 & $2,0.8 \%$ & - & 0.571 \\
\hline Ischemia- driven revascularization & 0 & $16,5.9 \%$ & - & 0.119 & 0 & $10,3.9 \%$ & - & 0.216 \\
\hline UAP & $2,1.9 \%$ & $30,11.2 \%$ & $6.73(1.61,28.2)$ & 0.009 & $1,0.8 \%$ & $14,5.4 \%$ & $6.0(0.79,45.6)$ & 0.084 \\
\hline Heart failure & $2,1.9 \%$ & 0 & - & 0.451 & $1,0.8 \%$ & $2,0.8 \%$ & _ & 0.891 \\
\hline
\end{tabular}

Values are presented as $\mathrm{n}, \%$. Rates are Kaplan-Meier estimates

OSA Obstructive Sleep Apnea, $C R$ complete revascularization, ICR Incomplete revascularization, $H R$ Hazard ratio, $C l$ confidence interval, $M A C C E s$ major adverse cardiovascular and cerebrovascular events, UAP unstable angina pectoris

cardiovascular events. OSA was reported to be associated with more multi-vessel disease, more severe coronary artery calcification, as well as more ST segment depression [20-22]. Our findings were consistent with the literature: higher frequency of three-vessel disease, two-vessel disease were observed in the OSA patients. We added further evidence that the OSA patients with ACS presented with more chronic total occlusion lesions, which finally led to more stent implantation comparing with the non-OSA patients. The possible mechanisms might include vascular endothelial injury, insulin resistance, sympathetic activation, neurohumoral changes, inflammation, oxidative stress, dyslipidemia through chronic intermittent hypoxia [23-25]. The disorders of metabolic function mentioned above ultimately induced or accelerated the process of coronary artery disease.
Several scoring systems have been developed to evaluate the complexity of coronary artery disease. Gensini score was deprived for each coronary stenosis on the basis of the degree of luminal narrowing and its topogtaphic importance [26]. SYNTAX score takes into account not only the luminal narrowing, but also other anatomic aspects, including bifurcation, trifurcation lesions, calcification, tortuosity, thrombus, and occlusions etc. Residual SYNTAX Score deriving from SYNTAX Score was used to quantify the residual burden of coronary artery disease after revascularization [12-14]. ICR was a surrogate marker of patients with more anatomically complex baseline disease. We found in the present study that ICR was more frequently observed in the OSA patients with ACS compared with those non- OSA. This implicates complex coronary lesion in the OSA patients and would predict worse prognostic events.
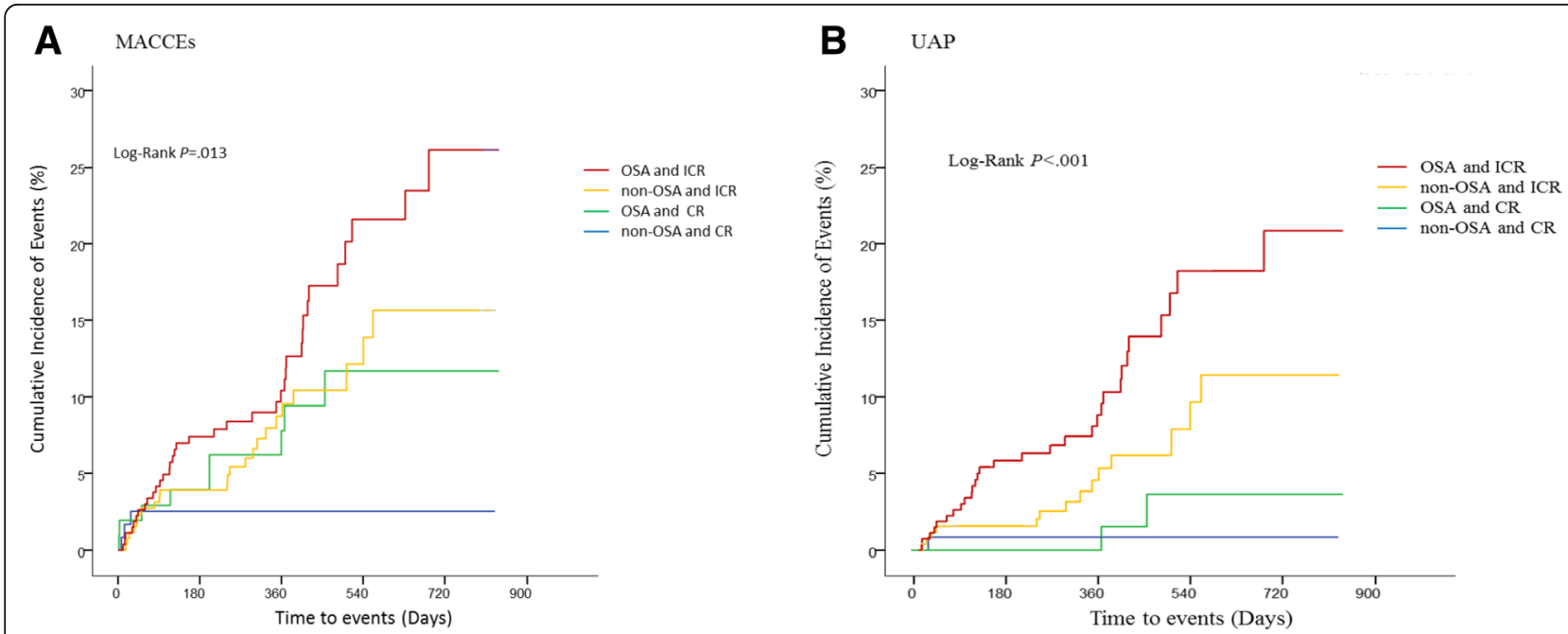

Fig. 3 Kaplan-Meier curve showing cumulative events at follow-up in subgroup analysis: (a) MACCEs in the OSA patients and ICR, non-OSA and ICR, OSA and CR, non-OSA patients and CR. $\mathbf{b}$ Unstable angina pectoris in the OSA patients and ICR, non-OSA and ICR, OSA and CR, non-OSA and $C R$. MACCES = major adverse cardiovascular and cerebrovascular events; UAP = unstable angina pectoris; OSA = Obstructive Sleep Apnea; nonOSA = non-Obstructive Sleep Apnea; $C R=$ complete revascularization; ICR = incomplete revascularization 
Table 3 Predictors of MACCE with Univariate and Multivariate Cox Regression Analyses, residual SYNTAX Score was drawn mandatory into the model

\begin{tabular}{lllll}
\hline & \multicolumn{2}{l}{ Univariate regression analysis } & & Multivariate regression analysis \\
\cline { 2 - 3 } & Hazard Ratio $(95 \% \mathrm{Cl})$ & $p$-value & & Hazard Ratio HR $(95 \% \mathrm{Cl})$ \\
\hline OSA & $1.68(1.04,2.72)$ & 0.034 & $1.65(1.02,2.67)$ & 0.044 \\
Residual Syntax Score & $1.03(1.0,1.05)$ & 0.014 & $2.04(1.09,3.8)$ & 0.025 \\
Diabetes mellitus & $1.82(1.14,2.9)$ & 0.012 & $1.59(1.0,2.57)$ & 0.056 \\
Arterial Hypertension & $1.69(0.98,2.91)$ & 0.059 & $1.37(0.78,2.4)$ & 0.273 \\
Age (years) & $1.03(1.0,1.05)$ & 0.029 & $1.02(0.99,1.04)$ & 0.231 \\
Female sex & $1.53(0.9,2.6)$ & 0.12 & $1.26(0.7,2.26)$ & 0.441 \\
\hline
\end{tabular}

$\mathrm{Cl}$ indicates confidence interval, OSA Obstructive Sleep Apnea

Previous studies have demonstrated that incomplete revascularization after either PCI or CABG was at a high risk of MACCEs in heterogeneous patients including ACS, STEMI, NSTEMI, multi-vessel disease and complex lesion [27-31]. However, studies evaluating the impact of rSS in patients with OSA and ACS have not yet been elucidated. We assessed for the first time the prognostic effect of rSS on clinical outcomes in a large prospective study. We found that ICR was associated with a 2-fold higher risk of MACCEs compared with CR in the OSA group. The OSA patients had a similar 1-year MACCEs as all the non-OSA if the revascularization was complete. Similarly, there was also no significant difference in the 1-year MACCEs rate in the OSA and the non-OSA patients if the revascularization was complete in both groups.

\section{Limitations}

There were several limitations in the study. Firstly, the non-randomized design of the study will preclude the conclusion. Secondly, the follow-up was relatively short to observe the incidence of long-term events. Thirdly, the sample size was relative small in subgroup analysis. Fourthly, rSS was based on angiographic interpretation that has inherent limitations [32]. However, rSS was assessed by consensus of well-trained analysts with good reproducibility. In addition, the strategy of PCI or CABG in the present study was decided by a heart team consisting of cardiac surgeons and cardiologists in one center, this reduces the potential confounder of operator discretion. Fifthly, residual SS was not calculated based on functional evaluation with fractional flow reserve, however, the latter has not been implemented routinely in multi-vessel disease in China.

\section{Conclusions}

ACS patients with OSA and ICR have a high rate of MACCEs at 1 year. In contrast, the prognosis of ACS patients with OSA but CR is favorable and similar to patients without non-OSA. Adequate level of revascularization is recommended to optimize clinical outcomes in ACS patients with OSA.

\section{Abbreviations}

ACS: Acute coronary syndrome; AHI: Apnea hypopnea index; CABG: Coronary artery bypass graft; CR: Complete revascularization; CTO: Chronic total occlusion; ICR: Incomplete revascularization; MACCEs: Major adverse cardiovascular and cerebrovascular events; OSA: Obstructive sleep apnea; PCl: Percutaneous coronary intervention; rSS: residual SYNTAX score; SS: SYNTAX score

\section{Acknowledgments}

Not applicable.

\section{Funding}

This work was supported by: International Science \& Technology Cooperation Program of China (2015DFA30160), Beijing Municipal Administration of Hospitals Clinical Medicine Development of Special Funding Support (ZYLX201710), National Natural Science Foundation of China (81800309), Beijing Municipal Administration of Hospitals' Youth Program (QML20170604). National Natural Science Foundation of China (81600209), Beijing Municipal Administration of Hospitals' Youth Program (QML20160605)

\section{Authors' contributions}

YZ and SY contributed to the SYNTAX analysis and manuscript writing. XW and JF contributed to the statistical analysis. SN and YW contributed to the design of the study and the manuscript writing. The authors thank Drs. Wen Hao, Guanqi Zhao, Shenghui Zhou, Aobo Li, Ruifeng Guo, Han Shi, and Zexuan Li for their help in data collection. All authors read and approved the final manuscript.

\section{Ethics approval and consent to participate}

The study was approved by the Institutional Review Board of Beijing Anzhen Hospital, Capital Medical University (2013025) and all patients provided written informed consent. All the authors consent to the publication of the manuscript.

\section{Competing interests}

The authors declare that they have no competing interests.

\section{Publisher's Note}

Springer Nature remains neutral with regard to jurisdictional claims in published maps and institutional affiliations.

\section{Author details}

${ }^{1}$ Emergency \& Critical Care Center, Beijing Anzhen Hospital, Capital Medical University, 2nd Anzhen Road, Chaoyang District, Beijing 100029, China. ${ }^{2}$ Department of Cardiology, Xinxiang Central Hospital, Xinxiang, Henan Province, People's Republic of China. ${ }^{3}$ Department of Otolaryngology Head \& Neck Surgery, Beijing Anzhen Hospital, Capital Medical University, 2nd Anzhen Road, Chaoyang District, Beijing 100029, China. 
Received: 9 August 2018 Accepted: 18 February 2019

Published online: 28 February 2019

\section{References}

1. Javaheri S, Barbe F, Campos-Rodriguez F, Dempsey JA, Khayat R, Javaheri S, Malhotra A, Martinez-Garcia MA, Mehra R, Pack Al, et al. Sleep Apnea. J Am Coll Cardiol. 2017:69:841-58.

2. Cowie MR. Sleep apnea: state of the art. Trends Cardiovasc Med. 2017; 27:280-9.

3. Alonso-Fernandez A, Garcia-Rio F, Arias MA, Hernanz A, de la Pena M, Pierola J, Barcelo A, Lopez-Collazo E, Agusti A. Effects of CPAP on oxidative stress and nitrate efficiency in sleep apnoea: a randomised trial. Thorax. 2009;64:581-6.

4. Jordan AS, McSharry DG, Malhotra A. Adult obstructive sleep apnoea. Lancet. 2014:383:736-47.

5. Somers VK, White DP, Amin R, Abraham WT, Costa F, Culebras A, Daniels S, Floras JS, Hunt CE, Olson $\sqcup$, et al. Sleep apnea and cardiovascular disease: an American Heart Association/american College Of Cardiology Foundation Scientific Statement from the American Heart Association Council for High Blood Pressure Research Professional Education Committee, Council on Clinical Cardiology, Stroke Council, and Council On Cardiovascular Nursing. In collaboration with the National Heart, Lung, and Blood Institute National Center on Sleep Disorders Research (National Institutes of Health). Circulation. 2008;118:1080-111.

6. Perk J, De Backer G, Gohlke H, Graham I, Reiner Z, Verschuren M, Albus C, Benlian P, Boysen G, Cifkova R, et al. European Guidelines on cardiovascular disease prevention in clinical practice (version 2012). The fifth joint task force of the European Society of Cardiology and Other Societies on cardiovascular disease prevention in clinical practice (constituted by representatives of nine societies and by invited experts). Eur Heart J. 2012:33:1635-701.

7. Lee CH, Sethi R, Li R, Ho HH, Hein T, Jim MH, Loo G, Koo CY, Gao XF, Chandra S, et al. Obstructive sleep apnea and cardiovascular events after percutaneous coronary intervention. Circulation. 2016;133:2008-17.

8. Tan A, Hau W, Ho H-H, Ghaem Maralani H, Loo G, Khoo S-M, Tai B-C, Richards AM, Ong P, Lee C-H. OSA and coronary plaque characteristics. Chest. 2014;145:322-30.

9. Barbe F, Duran-Cantolla J, Sanchez-de-la-Torre M, Martinez-Alonso M, Carmona C, Barcelo A, Chiner E, Masa JF, Gonzalez M, Marin JM, et al. Effect of continuous positive airway pressure on the incidence of hypertension and cardiovascular events in nonsleepy patients with obstructive sleep apnea: a randomized controlled trial. JAMA. 2012;307:2161-8.

10. Kohler M, Stradling JR. Mechanisms of vascular damage in obstructive sleep apnea. Nat Rev Cardiol. 2010;7:677-85.

11. Sianos G, Morel MA, Kappetein AP, Morice MC, Colombo A, Dawkins K, van den Brand M, Van Dyck N, Russell ME, Mohr FW, Serruys PW. The SYNTAX score: an angiographic tool grading the complexity of coronary artery disease. Eurolntervention. 2005;1:219-27.

12. Genereux P, Palmerini T, Caixeta A, Rosner G, Green P, Dressler O, Xu K, Parise H, Mehran R, Serruys PW, Stone GW. Quantification and impact of untreated coronary artery disease after percutaneous coronary intervention: the residual SYNTAX (synergy between PCI with Taxus and cardiac surgery) score. J Am Coll Cardiol. 2012:59:2165-74.

13. Farooq V, Serruys PW, Bourantas CV, Zhang Y, Muramatsu T, Feldman T, Holmes DR, Mack M, Morice MC, Stahle E, et al. Quantification of incomplete revascularization and its association with five-year mortality in the synergy between percutaneous coronary intervention with Taxus and cardiac surgery (SYNTAX) trial validation of the residual SYNTAX score * clinical perspective. Circulation. 2013;128:141-51.

14. Melina G, Angeloni E, Refice S, Benegiamo C, Lechiancole A, Matteucc M, Roscitano A, Bianchini R, Capuano F, Comito C, et al. Residual SYNTAX score following coronary artery bypass grafting. Eur J Cardiothorac Surg. 2017;51:547-53.

15. Qiu M, Li Y, Li J, Xu K, Jing Q, Dong S, Jin Z, Zhao P, Xu B, Han Y. Impact of six versus 12 months of dual antiplatelet therapy in patients with drugeluting stent implantation after risk stratification with the residual SYNTAX score: results from a secondary analysis of the I-LOVE-IT 2 trial. Catheter Cardiovasc Interv. 2017:89:565-73.

16. JL F. The design and analysis of clinical experiments. New York: Wiley; 1986

17. Sparrow D, Aloia M, Demolles DA, Gottlieb DJ. A telemedicine intervention to improve adherence to continuous positive airway pressure: a randomised controlled trial. Thorax. 2010;65:1061-6.
18. Barbe F, Duran-Cantolla J, Capote F, de la Pena M, Chiner E, Masa JF, Gonzalez M, Marin JM, Garcia-Rio F, de Atauri JD, et al. Long-term effect of continuous positive airway pressure in hypertensive patients with sleep apnea. Am J Respir Crit Care Med. 2010;181:718-26.

19. Schiza SE, Simantirakis E, Bouloukaki I, Mermigkis C, Kallergis EM, Chrysostomakis S, Arfanakis D, Tzanakis N, Vardas P, Siafakas NM. Sleep disordered breathing in patients with acute coronary syndromes. J Clin Sleep Med. 2012;8:21-6.

20. Abinader EG, Peled N, Sharif D, Lavie P. ST-segment depression during obstructive sleep apnea. Am J Cardiol. 1994;73:727.

21. Javadi HR, Jalilolghadr S, Yazdi Z, Rezaie Majd Z. Correlation between obstructive sleep apnea syndrome and cardiac disease severity. Cardiovasc Psychiatry Neurol. 2014;2014:631380.

22. Sorajja D, Gami AS, Somers VK, Behrenbeck TR, Garcia-Touchard A, LopezJimenez F. Independent association between obstructive sleep apnea and subclinical coronary artery disease. Chest. 2008;133:927-33.

23. Tan S, Liu X, Xu Y, Luo L, Zhou S, Gao Y. Serum high-density lipoprotein correlates with serum apolipoprotein $\mathrm{M}$ and $\mathrm{A} 5$ in obstructive sleep apnea hypopnea syndrome. Sleep Breath. 2017;21:37-44.

24. Karkinski D, Georgievski O, Dzekova-Vidimliski P, Milenkovic T, Dokic D. Obstructive sleep apnea and lipid abnormalities. Open Access Maced J Med Sci. 2017;5:19-22.

25. Guan J, Yi H, Zou J, Meng L, Tang X, Zhu H, Yu D, Zhou H, Su K, Yang M, et al. Distinct severity stages of obstructive sleep apnoea are correlated with unique dyslipidaemia: large-scale observational study. Thorax. 2016;71:347-55.

26. Erkan AF, Tanindi A, Kocaman SA, Ugurlu M, Tore HF. Epicardial adipose tissue thickness is an independent predictor of critical and complex coronary artery disease by Gensini and syntax scores. Tex Heart Inst J. 2016:43:29-37

27. Braga CG, Cid-Alvarez AB, Dieguez AR, Alvarez BA, Otero DL, Sanchez RO, Pena XS, Salvado VG, Trillo-Nouche R, Gonzalez-Juanatey JR. Prognostic impact of residual SYNTAX score in patients with ST-elevation myocardial infarction and multivessel disease: analysis of an 8-year all-comers registry. Int J Cardiol. 2017:243:21-6.

28. Diez-Delhoyo F, Sarnago Cebada F, Cressa LM, Rivera-Juarez A, Elizaga J, Fernandez-Aviles F. Prognostic Value of the Residual SYNTAX Score in Octogenarian Patients With Non-ST-elevation Acute Coronary Syndrome. Rev Esp Cardiol (Engl Ed). 2016;69:217-9.

29. Song Y, Xu JJ, Tang XF, Ma YL, Yao Y, He C, Wang HH, Liu R, Xu N, Jiang P, et al. Usefulness of the residual SYNTAX score to predict long term outcome in acute coronary syndrome patients underwent percutaneous coronary intervention. Zhonghua Yi Xue Za Zhi. 2017;97:502-7.

30. Witberg G, Lavi I, Assali A, Vaknin-Assa H, Lev E, Kornowski R. The incremental impact of residual SYNTAX score on long-term clinical outcomes in patients with multivessel coronary artery disease treated by percutaneous coronary interventions. Catheter Cardiovasc Interv. 2015;86:3-10.

31. Schwietz T, Spyridopoulos I, Pfeiffer S, Laskowski R, Palm S, DER S, Jens K, Zeiher AM, Schachinger V, Fichtlscherer S, Lehmann R. Risk stratification following complex PCl: clinical versus anatomical risk stratification including "post PCI residual SYNTAX-score" as quantification of incomplete revascularization. J Interv Cardiol. 2013;26:29-37.

32. Tonino PA, Fearon WF, De Bruyne B, Oldroyd KG, Leesar MA, Ver Lee PN, Maccarthy PA, Van't Veer M, Pijls NH. Angiographic versus functional severity of coronary artery stenoses in the FAME study fractional flow reserve versus angiography in multivessel evaluation. J Am Coll Cardiol. 2010;55:2816-21.

\section{Ready to submit your research? Choose BMC and benefit from}

- fast, convenient online submission

- thorough peer review by experienced researchers in your field

- rapid publication on acceptance

- support for research data, including large and complex data types

- gold Open Access which fosters wider collaboration and increased citations

- maximum visibility for your research: over $100 \mathrm{M}$ website views per year

At $\mathrm{BMC}$, research is always in progress.

Learn more biomedcentral.com/submissions 